\title{
Cardiovascular risk stratification in diabetic patients: Is all in METS?
}

\author{
Mario Petretta, MD, ${ }^{a}$ Wanda Acampa, MD, PhD, ${ }^{b}$ and Alberto Cuocolo, $\mathrm{MD}^{\mathrm{c}}$ \\ a Department of Translational Medical Sciences, University Federico II, Naples, Italy \\ ${ }^{b}$ Institute of Biostructure and Bioimaging, National Council of Research, Naples, Italy \\ c Department of Advanced Biomedical Sciences, University Federico II, Naples, Italy
}

Received May 28, 2014; accepted May 28, 2014

doi: $10.1007 / \mathrm{s} 12350-014-9931-3$

\section{See related article, pp. 1132-1143}

Diabetes mellitus is a planetary growing problem and the costs to society are high and increasing. There are an estimated 382 million people living with diabetes in 2013 and this number is expected to increase to 592 millions by 2035. ${ }^{1}$ Landmark epidemiological studies reported that diabetes is associated with a marked increase in the risk of coronary artery disease (CAD). ${ }^{2,3}$ As cardiovascular diseases account for about $65 \%$ of diabetes-related mortality, toward the end of the twentieth century the American Heart Association defined diabetes as coronary heart disease equivalent. ${ }^{4}$ Cardiac autonomic neuropathy and diabetic cardiomyopathy are the other two forms of cardiac involvement in diabetic subjects. ${ }^{5}$

\section{STRESS PERFUSION IMAGING IN DIABETIC PATIENTS}

The objective of non-invasive testing diabetic patients is the detection of occult CAD as well as prognostic risk stratification. For these purposes, among the non-invasive imaging modalities, stress myocardial perfusion single-photon emission computed tomography (MPS) is widely used and seems valuable also in asymptomatic diabetic patients. ${ }^{6,7}$ The prospective multinational ischemia assessment with exercise imaging in

Reprint requests: Alberto Cuocolo, MD, Advanced Biomedical Sciences, University Federico II, Via Pansini 5, 80131 Naples, Italy; cuocolo@unina.it

J Nucl Cardiol 2014;21:1144-47.

$1071-3581 / \$ 34.00$

Copyright (c) 2014 American Society of Nuclear Cardiology. asymptomatic diabetes from 12 sites in Asia, Africa, and Latin America found that a higher proportion of the diabetic patients enrolled had MPS abnormalities suggestive of silent ischemia compared with controls $(26 \%$ vs. $14 \% ; P<.001){ }^{7}$ Of the participants with MPS ischemia, $17 \%$ had ischemic signs on electrocardiography (ECG), whereas $10 \%$ of those without ischemia had an ischemic ECG. In a multivariable model, diabetes was independently associated with abnormal MPS $(P<.005)$. Women were less likely to have ischemia on MPS than men and concordance between ECG and MPS was much worse in women. In this study of nonselected individuals from developing countries, ${ }^{7}$ the prevalence of ischemia by MPS was higher than was seen in the Detection of Ischemia in Asymptomatic Diabetes (DIAD) trial. ${ }^{8}$ In this latter study, the prevalence of ischemia was only $6 \%$. At follow-up, the DIAD investigators found low event rate with similar outcomes in participants who were randomized to screening MPS vs no screening, ${ }^{9}$ hypothesizing that intensive medical treatment may overcome the possible benefit from screening. ${ }^{10}$ However, higher prevalence of silent ischemia by MPS in diabetic subjects has been reported in other studies. $^{11,12}$

\section{IMPROVEMENT IN RISK ESTIMATION WHEN AN ADDITIONAL TEST IS ADDED TO A STANDARD MODEL}

Different methods for quantifying the improvement in risk estimation when an additional test is added to a standard risk prediction model have recently been introduced. ${ }^{13}$ Net reclassification improvement (NRI) has been adopted in diabetic patients with suspected or known CAD to evaluate the extent to which adding MPS imaging findings to a model based on traditional risk factors and ECG stress test data correctly reclassifies the risk of severe CAD and of subsequent cardiac events during a long-term follow-up. ${ }^{6,14-17}$ In the Impact of 
Inducible Ischemia by Stress SPECT (IDIS) trial, risk estimates for a coronary heart disease event were categorized in 822 consecutive diabetic patients as $0 \%$ to $<3 \%, 3 \%$ to $<5 \%$, and $\geq 5 \%$ per year using two Cox proportional hazards models. ${ }^{16,17}$ Model 1 used traditional coronary heart disease risk factors and ECG stress test data and model 2 used these variables plus MPS imaging data. Cardiac death, myocardial infarction, and unstable angina requiring coronary revascularization were the outcome measures. Model 2 improved risk prediction compared to model 1 (NRI 0.25; $P<.001$ ). Overall, 301 patients were reclassified to a higher risk category, with an event rate of $28 \%$, and 26 to a lower risk category, with an event rate of $15 \%$. Among patients at $3 \%$ to $<5 \%$ risk, $53 \%$ were reclassified at higher risk and $25 \%$ at lower risk (NRI $0.42 ; P<.05$ ). The cost per NRI was $\$ 880.80$ for MPS imaging as compared to an outpatient visit with an ECG stress test. Thus, the addition of MPS imaging data to a prediction model based on traditional risk factors and ECG stress test data significantly improved coronary heart disease risk classification in patients with diabetes. ${ }^{17}$

\section{PROGNOSTIC VALUE OF EXERCISE LEVEL IN DIABETIC PATIENTS}

Exercise stress testing is largely used as noninvasive, cost-effective method to evaluate CAD. Exercise stress test provides other valuable diagnostic and prognostic data, beyond ST-segment depression. ${ }^{18}$ These variables include exercise capacity, chronotropic response, heart rate recovery, blood pressure response, and scores such as the Athens QRS score and the Duke Treadmill Score. ${ }^{19,20}$ However, among the various parameters and scores introduced as prognostic markers by the exercise test, one of the most important is the functional capacity assessed as metabolic equivalents (METS). ${ }^{21}$ In the current issue of the Journal, Padala et $\mathrm{al}^{22}$ present data obtained analyzing retrospectively 14,849 consecutive patients $(3,654$ diabetics and 11,195 non-diabetics) undergoing exercise stress, combined exercise and pharmacologic stress, and pharmacologic stress MPS from 1995 to 2005 at a single tertiary care center. Diabetic and non-diabetic patients were categorized into three groups based on METS achieved: $\geq 5$ METS, <5 METS, and pharmacologic stress groups. Annualized event rate for the composite end point of non-fatal myocardial infarction and cardiac death was calculated over a mean follow-up period of $2.4 \pm 1.4$ years with a maximum of 6 years. In moderate-severe perfusion abnormality category, diabetic patients who were able to achieve $\geq 5$ METS had significantly lower annualized event rate compared to diabetic patients who were unable to achieve $\geq 5$ METS
(3\% vs. $5.5 \% ; P<.05)$, and non-diabetic patients unable to achieve $\geq 5$ METS (3\% vs. $4.8 \%$; $P<.001$ ). Diabetic patients who achieved a high workload $(\geq 10$ METS) had a low annualized event rate of $0.9 \%$. After adjustment for cardiovascular risk factors, the percentage decrease in cardiac event rate for every 1-MET increment in exercise capacity was $10 \%$ in the overall cohort, $12 \%$ in diabetic group, and $8 \%$ in non-diabetic group. Thus, despite significant perfusion defects, diabetic patients who achieve $\geq 5$ METS during stress MPS have significantly reduced risk for future cardiac events. The findings of Padala et $\mathrm{al}^{22}$ support the view that exercise capacity obtained during MPS is a surrogate for outcomes also among diabetic patients undergoing nuclear stress testing. When a concept gains general acceptance, its underlying basis may no longer be questioned. Thus, the limitations intrinsic in its assumption are ignored, and the possibility of misusing the concept may increase. There are some limitations in using METS. The definition of a MET is problematic when applied for specific persons because it is not clear how or when the 1 MET value of $3.5 \mathrm{~mL} \mathrm{O}_{2} \mathrm{~kg}^{-1}$ $\min ^{-1}$ (or $1 \mathrm{kcal} \mathrm{kg}^{-1} \mathrm{~h}^{-1}$ ) was derived. ${ }^{23}$ Yet, it is a common practice for researchers and practitioners to use the MET values to judge the energy cost of different activities. $^{24}$ The conventional MET is not specifically a constant that equates resting values. Byrne et $\mathrm{al}^{25}$ measured MET level for moderate-intensity walking in an overweight cohort and found an average value of $4.6 \pm 0.5,22 \%$ higher than the compendium value of 3.8. Previous studies by Bourque et $\mathrm{al}^{26}$ suggested that in patients who exercise $>10$ METS, MPS adds little to the diagnosis of $\mathrm{CAD}$ and to risk stratification. ${ }^{27}$ Noteworthy, the difference in threshold used by Padala et $\mathrm{al}^{22}$ (5 METS) and Bourque et $\mathrm{al}^{26,27}$ (10 METS) seems not negligible as these thresholds relate to stage 1 and stage 3 of the Bruce protocol, respectively. Remarkably, the prevalence of ischemia and its prediction of events are unclear in outpatients with diabetes mellitus in the modern era of intensive medical management. Bourque et $\mathrm{al}^{28}$ recently sought to identify the prevalence of ischemia, subsequent cardiac events, and impact of sex, stress type, and symptom status on these findings in a cohort of stable outpatients with diabetes mellitus referred for MPS. Known CAD was present in $40.3 \%$ of subjects and $29 \%$ were asymptomatic at the time of stress testing. In this cohort of stable outpatients with diabetes mellitus, independent predictors of cardiac death/non-fatal myocardial infarction were known CAD, pharmacological stress, and MPS ischemia. In the subgroup of 200 patients who underwent exercise stress, there were 1.6-fold and 3.8-fold increases in any and $\geq 10 \%$ of the left ventricle ischemia in those unable to reach 10 METS of exercise workload; however, these 
differences were not statistically significant. There were no significant differences in summed stress or rest scores by symptom status in subjects undergoing pharmacological stress. However, asymptomatic patients undergoing exercise stress were more likely to achieve a high exercise workload of $\geq 10$ METS (58.3\% vs. $23.1 \%$; $P=.007)$, and to have a summed stress score $>3$ ( $29.7 \%$ vs. $12.9 \% ; P=.011)$ despite being able to attain a higher workload. Noteworthy, in this study nearly one third of patients with events had a normal MPS, indicating a need for improved risk stratification. ${ }^{28}$ Thus, the relationship among METS, stress-induced ischemia, and prognosis in diabetic subjects remains unclear. In another recent study, Duvall et $\mathrm{al}^{29}$ showed that a provisional injection protocol defined as age $<65$, normal rest ECG, no history of CAD, and high level exercise ( $\geq 10$ METS) with a negative ECG response and no symptoms has a very low 5-year all-cause mortality and low yield of MPS. If adopted, this protocol would decrease radiation exposure and save time and health care costs without jeopardizing prognosis. Based on the available retrospective data regarding the very low prevalence of significant ischemia and excellent prognosis for patients achieving $\geq 10$ METS without ischemic ST depression, including those reported in this issue by Padala et $\mathrm{al}^{22}$ this provisional injection protocol deserves further consideration and prospective evaluation in randomized controlled study. ${ }^{30}$ This approach might best identify which patients do not require MPS at the outset for evaluation of symptoms suggestive of CAD. Indeed, a non-imaging exercise ECG stress test could be performed alone as the first test in patients prospectively identified as having good functional capacity and deemed at low or low-intermediate pre-test probability for CAD.

\section{Conflict of interest}

The authors have indicated that they have no financial conflict of interest.

\section{References}

1. Guariguata L, Whiting DR, Hambleton I, Beagley J, Linnenkamp U, Shaw JE. Global estimates of diabetes prevalence for 2013 and projections for 2035. Diabetes Res Clin Pract 2014;103:137-49.

2. Pyörälä K, Laakso M, Uusitupa M. Diabetes and atherosclerosis: An epidemiologic view. Diabetes Metab Rev 1987;3:463-524.

3. Stamler J, Vaccaro O, Neaton JD, Wentworth D. Diabetes, other risk factors, and 12-year cardiovascular mortality for men screened in the Multiple Risk Factor Intervention Trial. Diabetes Care 1993;16:434-44.

4. Grundy SM, Benjamin IJ, Burke GL, Chait A, Eckel RH, Howard BV, et al. Diabetes and cardiovascular disease: A statement for healthcare professionals from the American Heart Association. Circulation 1999;100:1134-46.

5. Paolillo S, Rengo G, Pagano G, Pellegrino T, Savarese G, Femminella GD, et al. Impact of diabetes on cardiac sympathetic innervation in patients with heart failure: ${ }^{123} \mathrm{I}$ meta-iodobenzylguanidine ( ${ }^{123} \mathrm{I}$ MIBG) scintigraphic study. Diabetes Care 2013;36:2395-401.

6. Acampa W, Petretta M, Daniele S, Del Prete G, Assante R, Zampella $\mathrm{E}$, et al. Incremental prognostic value of stress myocardial perfusion imaging in asymptomatic diabetic patients. Atherosclerosis 2013;227:307-12.

7. Hage FG, Lusa L, Dondi M, Giubbini R. Iskandrian AE; IAEA Diabetes Investigators. Exercise stress tests for detecting myocardial ischemia in asymptomatic patients with diabetes mellitus. Am J Cardiol 2013;112:14-20.

8. Wackers FJ, Young LH, Inzucchi SE, Chyun DA, Davey JA, Barrett EJ, et al. Detection of silent myocardial ischemia in asymptomatic diabetic subjects: The DIAD study. Diabetes Care 2004;27:1954-61.

9. Young LH, Wackers FJ, Chyun DA, Davey JA, Barrett EJ, Taillefer R, et al. Cardiac outcomes after screening for asymptomatic coronary artery disease in patients with type 2 diabetes: The DIAD study: A randomized controlled trial. JAMA 2009;301:1547-55.

10. Bansal S, Wackers FJ, Inzucchi SE, Chyun DA, Davey JA, Staib $\mathrm{LH}$, et al. 5-year outcomes in high-risk participants in the Detection of Ischemia in Asymptomatic Diabetics (DIAD) study: A post hoc analysis. Diabetes Care 2011;34:204-9.

11. Prior JO, Monbaron D, Koehli M, Calcagni ML, Ruiz J, Bischof Delaloye A. Prevalence of symptomatic and silent stress-induced perfusion defects in diabetic patients with suspected coronary artery disease referred for myocardial perfusion scintigraphy. Eur $\mathbf{J}$ Nucl Med Mol Imaging 2005;32:60-9.

12. Rajagopalan N, Miller TD, Hodge DO, Frye RL, Gibbons RJ. Identifying high-risk asymptomatic diabetic patients who are candidates for screening stress single-photon emission computed tomography imaging. J Am Coll Cardiol 2005;45:43-9.

13. Petretta M, Cuocolo A. Prediction models for risk classification in cardiovascular disease. Eur $\mathrm{J}$ Nucl Med Mol Imaging 2012;39:1959-69.

14. Petretta M, Acampa W, Daniele S, Petretta MP, Nappi C, Assante $\mathrm{R}$, et al. Transient ischemic dilation in SPECT myocardial perfusion imaging for prediction of severe coronary artery disease in diabetic patients. J Nucl Cardiol 2013;20:45-52.

15. Petretta M, Acampa W, Daniele S, Petretta MP, Plaitano M, Cuocolo A. Transient ischemic dilation in patients with diabetes mellitus: Prognostic value and effect on clinical outcome after coronary revascularization. Circ Cardiovasc Imaging 2013;6:90815.

16. Petretta M, Acampa W, Evangelista L, Daniele S, Ferro A, Cuocolo A, Impact of Inducible Ischemia by Stress SPECT (IDIS) Investigators. Impact of inducible ischemia by stress SPECT in cardiac risk assessment in diabetic patients: Rationale and design of a prospective, multicenter trial. J Nucl Cardiol 2008;15:100-4.

17. Acampa W, Petretta M, Evangelista L, Daniele S, Xhoxhi E, De Rimini ML, et al. Myocardial perfusion imaging and risk classification for coronary heart disease in diabetic patients. The IDIS study: A prospective, multicentre trial. Eur J Nucl Med Mol Imaging 2012;39:387-95.

18. Sharma K, Kohli P, Gulati M. An update on exercise stress testing. Curr Probl Cardiol 2012;37:177-202.

19. Michaelides AP, Tousoulis D, Raftopoulos LG, Antoniades C, Tsiachris D, Stefanadis CI. The impact of novel exercise criteria and indices for the diagnostic and prognostic ability of exercise testing. Int J Cardiol 2010;143:119-23. 
20. Mark DB, Hlatky MA, Harrell FE Jr, Lee KL, Califf RM, Pryor DB. Exercise treadmill score for predicting prognosis in coronary artery disease. Ann Intern Med 1987;106:793-800.

21. Morris CK, Ueshima K, Kawaguchi T, Hideg A, Froelicher VF. The prognostic value of exercise capacity: A review of the literature. Am Heart J 1991;122:1423-31.

22. Padala S, Ghatak A, Sandeep P, Deborah K, Donna P, Heller G. Cardiovascular risk stratification in diabetic patients following stress single photon emission computed tomography myocardial perfusion imaging: The impact of achieved exercise level. J Nucl Cardiol 2014. doi:10.1007/s12350-014-9986-1.

23. Jette M, Sidney K, Blumchen G. Metabolic equivalents (METS) in exercise testing, exercise prescription, and evaluation of functional capacity. Clin Cardiol 1990;13:555-65.

24. Ainsworth BE, Haskell WL, Whitt MC, Irwin ML, Swartz AM, Strath SJ, et al. Compendium of physical activities: An update of activity codes and MET intensities. Med Sci Sports Exerc 2000;32:498-516.

25. Byrne NM, Hills AP, Hunter GR, Weinsier RL, Schutz Y. Metabolic equivalent: One size does not fit all. J Appl Physiol 2005;99:1112-9.
26. Bourque JM, Holland BH, Watson DD, Beller GA. Achieving an exercise workload of $\geq 10$ metabolic equivalents predicts a very low risk of inducible ischemia: Does myocardial perfusion imaging have a role? J Am Coll Cardiol 2009;54:538-45.

27. Bourque JM, Charlton GT, Holland BH, Belyea CM, Watson DD, Beller GA. Prognosis in patients achieving $\geq 10$ METS on exercise stress testing: Was SPECT imaging useful? J Nucl Cardiol 2011;18:230-7.

28. Bourque JM, Patel CA, Ali MM, Perez M, Watson DD, Beller GA. Prevalence and predictors of ischemia and outcomes in outpatients with diabetes mellitus referred for single-photon emission computed tomography myocardial perfusion imaging. Circ Cardiovasc Imaging 2013;6:466-77.

29. Duvall WL, Levine EJ, Moonthungal S, Fardanesh M, Croft LB, Henzlova MJ. A hypothetical protocol for the provisional use of perfusion imaging with exercise stress testing. J Nucl Cardiol 2013;20:739-47.

30. Beller GA, Bateman TM. Provisional use of myocardial perfusion imaging in patients undergoing exercise stress testing: A worthy concept fraught with challenges. J Nucl Cardiol 2013;20:711-4. 